\title{
Digital Video Watermarking based on Different Wavelet Transform
}

\author{
Dhanashree S. Shedge \\ PG Student, Department of Computer, \\ Yadavrao Tasgaonkar \\ College of Engineering \& Management, \\ (YTCEM), Karjat, India
}

\author{
Aarti G. Ambekar \\ Assistant Professor, Department of EXTC, \\ DJ Sanghvi College of Engineering, (DJSCE), \\ Mumbai, India
}

\begin{abstract}
In one's everyday life, web is developing and turned into an imperative part. Digital content can easily be downloaded, duplicated or altered. Digital content can be secured by number of ways. Digital Watermarking is one of the strategies for the insurance of Digital Content. Digital Watermarking is a plan of copyright protection in which a watermark data is embedded into cover image in such a way that the watermark data is not detected and it is invisible. A watermark will be an intellectual property rights is in the form of image, audio, text, numbers etc emerged into images, video files, audio files and other multimedia data having assured methods, approaches, algorithms. To protect the authentication and authorization rights, different wavelet transform based techniques have been proposed to making the watermarked data more robust, imperceptible and secure. In this work, watermarking has been done using SVD method, and comparison of Discrete wavelet transform (DWT) and Lifting wavelet transform(LWT). The results of the scheme are compared the imperceptibility and the robustness of the video frame, on the basis of the parameters such as, correlation coefficient (CC), peak signal to noise ratio (PSNR) and structural similarity index (SSIM ).
\end{abstract}

\section{General Terms}

Digital watermarking, Video watermarking.

\section{Keywords}

Discrete Wavelet Transform, Lifting Wavelet Transform, Singular Value Decomposition, Copyright Protection.

\section{INTRODUCTION}

Digital watermarking is referred as an advanced watermarking otherwise called watermark insertion or watermark implanting, addresses the system for embeddings information into sight and sound information additionally called unique media or spread media e.g. content, sound, picture, video. The inserted data or watermark can be a serial number or arbitrary number grouping, proprietorship identifiers, copyright messages, control signals, exchange dates, data about the makers of the work, bi-level or dim level pictures, content or other advanced information designs. In the writing expansive number of content, picture, sound and video watermarking calculations can be found. These calculations change the first media to produce the watermarked media. There might be no or minimal recognizable contrasts between the unique media and the watermarked media.

Video-watermarking refers to the method of embedding the data or information into the frames of video and extract the procedure to retrieve the data from the reconstructed video. Clearly any picture watermarking strategy can be stretched out to watermark recordings, yet in actuality video watermarking systems need to meet different difficulties than that in picture watermarking plans, for example, huge volume of naturally repetitive information between edges, the unbalance between the movement and still districts, ongoing prerequisites in the video TV and so on. Watermarked video successions are in particular helpless to privateer assaults, for example, outline averaging, outline swapping, factual investigation, advanced simple (Advertisement/DA) change, and lossy compressions. Video watermarking applications can be gathered as security related like Copy control, fingerprinting, proprietorship distinguishing proof, validation, decrease resistance and so forth or worth included applications like legacy framework improvement, database connecting, video labelling, computerized video telecast checking, Media Span and so on. Aside from vigour, dependability, intangibility, common sense, and video watermarking calculations should likewise address issues, for example, restricted recognition, ongoing calculation manysided quality, synchronization, recuperation, impacts of coasting point representation, power dispersal and so on. As indicated by the working space, video watermarking systems are ordered in pixel area and change area methods. In pixel space the watermark is installed in the source video by straightforward expansion or bit substitution of chosen pixel positions. The primary points of interest of utilizing pixel space procedures are that they are theoretically easy to comprehend and the time multifaceted nature of these methods are low which supports constant executions. In any case, these methods for the most part needs in giving sufficient power and impalpability necessities. In change space techniques, the host sign is changed into an alternate space and watermark is inserted in particular coefficients. Ordinarily utilized change systems are discrete cosine change (DCT) and discrete wavelet change (DWT). The primary point of interest of the changed space watermarking is the simple relevance of extraordinary changed space properties.

\section{DISCRETE WAVELET TRANSFORM}

As for schemes working in other transform domains, the watermark is usually given by a pseudo-random 2-D pattern. Both the image and watermark are decomposed using a 2-D wavelet transform and in each subband of the image a weighted rendition of the watermark is included. Watermark interpreting is,, as usual, based on a normalized correlation between the evaluation of the implanted watermark and the watermark itself. Various wavelet-based schemes have been proposed (Raghavendra K. and Chetan K.R, [1]; Sanjana Sinha, Prajnat Bardhan, Swarnali Pramanick, Ankul Jagatramka, Dipak K. Kole, Aruna Chakraborty [2], ; Tamanna Tabassum and S.M. Mohidul Islam [3]; Himanshu 
Agarwal,Rakesh Ahuja,S.S.Bedi [4]; The differences between the schemes usually lie in the way the watermark is weighted in order to decrease visual artifacts.

Raghavendra K. Chetan K.R [1] proposed a Robust watermarking approach based on wavelet transformation. In this method a robust Discrete Wavelet Transform (DWT)based visually impaired advanced video watermarking scheme with mixed watermarks taking into account scene changes has been proposed for confirmation of advanced video, which embeds different parts of a single watermark into various scenes of a video. The video watermarking algorithm is robust against the attacks of frame dropping, averaging and pressure, which are considered as a few of the common types of attacks applied particularly on the video and because of the utilization of DWT it can likewise withstand geometrical attacks making the watermark perceptually invisible. Furthermore, it permits blind recovery of inserted watermark which does not need the original video. The proposed calculation has been differentiated DWT based watermarking plan and is found to exhibit better robustness.

A Comprehensive approach for watermarking digital video is introduced by Sanjana Sinha, Prajnat Bardhan, Swarnali Pramanick, Ankul Jagatramka, Aruna Chakraborty [2], In this paper a hybrid digital video watermarking scheme has been presented . PCA helps in decreasing correlation among the wavelet coefficients got from wavelet decay of each video frame consequently scattering the watermark bits into the uncorrelated coefficients. The cover frame are first decomposed using DWT and the binary watermark is embedding the foremost parts of the low frequency wavelet coefficients. The imperceptible high bit rate watermark implanted is strong against different assaults that can be conveyed out on the watermarked video, such as filtering, contrast change, commotion expansion and geometric assaults..

Tamanna Tabassum, S.M. Mohidul Islam [3] presented an advanced video watermarking strategy in light of indistinguishable casing extraction in 3-Level Discrete Wavelet Transform (DWT). In the proposed strategy, first the host video is isolated into video shots. At that point from every video shot one video outline called indistinguishable casing is chosen for watermark implanting. Each indistinguishable casing is disintegrated into 3-level DWT, then select the higher subband coefficients to implant the watermark and the watermark are adaptively inserted to these coefficients and accordingly ensure the perceptual intangibility of the watermark. For watermark recognition, the connection between's the watermark signal and the watermarked video is contrasted and a limit esteem got from inserted watermark signal. The trial results show that the watermarking technique has solid power against some regular assaults, for example, trimming, Gaussian commotion including, Salt and pepper clamor including, outline dropping and outline including.

Himanshu Agarwal,Rakesh Ahuja,S.S.Bedi [4] proposed a Hybrid computerized video watermarking plan in light of discrete wavelet change and solitary quality disintegration. Not at all like the most existing watermarking plans, the utilized watermark is a dim scale picture rather than a paired watermark. The watermark is installed in the first video outlines by initially changed over it into $\mathrm{YCbCr}$ shading space and than decaying the luminance part (Y segment) into four sub-groups utilizing discrete wavelet change lastly the particular estimations of LL sub-band are formed perceptually by solitary estimations of watermark picture. The test result demonstrates a tradeoff amongst subtlety and strength against purposeful assaults, for example, revolution, trimming, histogram extending, JPEG pressure on individual edges, video pressure and unexpected assaults like edge swapping, outline averaging, outline insertion and distinctive sorts of clamor expansion. Predominance of the proposed plan is completed by correlation with existing plans to uncover its proficiency for functional applications.

\section{LIFTING WAVELET TRANSFORM}

Souvik Sarkar, K. Senthilkumar [5] introduced a new highly secured Digital Watermarking Algorithm especially for Binary Watermark utilizing Lifting Wavelet Transform (LWT) and Singular Value Decomposition (SVD). Its main motive is to keep watermark in place to the first image furthermore to build the data embedding \& extraction rate. In this only the LL sub-band (containing an estimate of the image) of the cover image obtained from Lifting Wavelet Transform and then apply Singular Value Decomposition to insert the binary watermark. Moreover due to usage of lifting based wavelet transform and SVD, the implementation of hardware is better.

Swanirbhar Majumder, Tirtha Sankar Das, Souvik Sarkar and Subir Kumar Sarka [6] proposed Fragile image watermarking using the discrete lifting based wavelet transform and the singular value decomposition (SVD) are used in this scheme. The former for the carrier or the image to be authenticated, while the last for the logo which is installed in the cover image. The distribution of SVD compressed pixel values are conveyed in the wavelet region considering a pseudorandom grouping. This has been observed to test the integrity of the stego image and its its confirmation. In addition because of utilization of lifting based change and SVD the equipment implementability is better. This SVD and lifting based discrete wavelet transform based system of fragile watermarking fulfils its motivation of validating the carrier image, as observed for the attacks considered. The region of attack or tampering can be perceived by survey the adjustments in the removed logo and contrasting it with the original. Dissimilar to other mainstream strategies for watermarking this is not a hybrid scheme, involving two transforms on the carrier itself. Or maybe one of the changes follow up on the carrier and the other on the logo to be inserted, independently. The fundamental key element here of both the transforms is that, both of them can be easily executed in equipment. This is an essential point of preference, as present-day frameworks are more arranged for rate and scaling down. So with the feature of less demanding equipment implementability, both of these are taken into account.

Pragya Agarwal, Arvind Kumar, Ankur Choudhary [7] proposed LWT(Lifting Wavelet Transform) and SVD(Singular Value Decomposition) is used to design a video watermarking technique. The video is divided into different scenes, and the last frame of each scene is selected for embedding watermark. A gray scale watermark is selected for this purpose. Each RGB video frame is converted into $\mathrm{YCbCr}$ format, and then luminance part $(\mathrm{Y})$ is taken for inserting the watermark. The LWT is applied on $\mathrm{Y}$ component of the video frame to decompose it into four sub parts (LL, LH, HL, HH). Then SVD is applied on the LL sub part which decomposes it into $\mathrm{U}, \mathrm{S}$, and $\mathrm{V}$ components. Finally watermark is also decomposed using SVD to get $\mathrm{Sw}, \mathrm{Uw}$ and $\mathrm{Vw}$. Then $\mathrm{S}$ 
component of the cover video frame is modified using $\mathrm{Sw}$ component of the watermark to embed the watermark in the cover frame. Then Inverse SVD and Inverse LWT are applied to get the final watermarked video frame. The Imperceptibility, time complexity and robustness of the watermarking method is checked by applying some intentional attacks on the watermarked video frame.

\section{SINGULAR VALUE DECOMPOSITION}

The Singular Value Decomposition (SVD) is also gaining interest in watermarking. The SVD for square matrices was discovered 51 independently by Beltrami in 1873 and Jordan in 1874 and extended to rectangular matrices by Eckart and Young in the year 1930s. It was not utilized as a computational tool until the 1960s because of the need for sophisticated numerical strategies. In later years, Gene Golub demonstrated its usefulness and feasibility as a tool in a variety of applications (Kahaner et al., 1989). SVD is one of the most useful tools of linear algebra with several applications in image compression (Waldemar and Ramstad, 1997; Aase et al., 1999) and other signal processing fields (Konstantinides et al., 1997; Karkarala and Ogunbona, 2001). Because of its robust nature and the property of maintaining visual quality, SVD in watermarking has been most exploited (Prathik P, Rahul Krishna, Rahul Ajay Nafde, Shree Darshan K[8]; Bhavna Goel Charu Agarwal [9].

Prathik P, Rahul Krishna, Rahul Ajay Nafde, Shree Darshan $\mathrm{K}$ [8], proposed an Adaptive Blind Video Watermarking based on DWT-SVD techniques. Data Exchange in the form of multimedia has seen a tremendous growth in the previous decade along these lines requiring a requirement for better security and protection for proprietary rights. The proposed ideas incorporate Adaptive Frame Selection utilizing SDBPSO to guarantee the watermarks have minimum inconvenient impacts on the video overall.. The integrity of the video is accepted utilizing the Peak Signal to Nose Ratio . The robustness is also tested by subjecting the video to a few standard assaults, such as rotation, cropping, image shifting, histogram equalization and. Bit Error Rate (BER) is also used to determine the efficiency of the system in retaining the watermark.

Bhavna Goel Charu Agarwal [9] presents a Novel fast and robust video watermarking scheme for RGB uncompressed AVI video file in discrete wavelet transform (DWT) area utilizing singular value decomposition (SVD). The binary watermark area unit embedded among the singular values of the LL3 sub-band of the video frames. The resultant marked video displays great quality. The high computed PSNR values indicate that the quality of the marked and assaulted video is great. The low bit error rate and high correlation between's the removed and inserted watermark. Time unpredictability shows that the proposed scheme is appropriate for real time application. For embedding and extraction procedure, the proposed algorithm are very much upgraded. The algorithm is robust and demonstrates a change over other comparable reported strategies.

\section{PROPOSED METHOD}

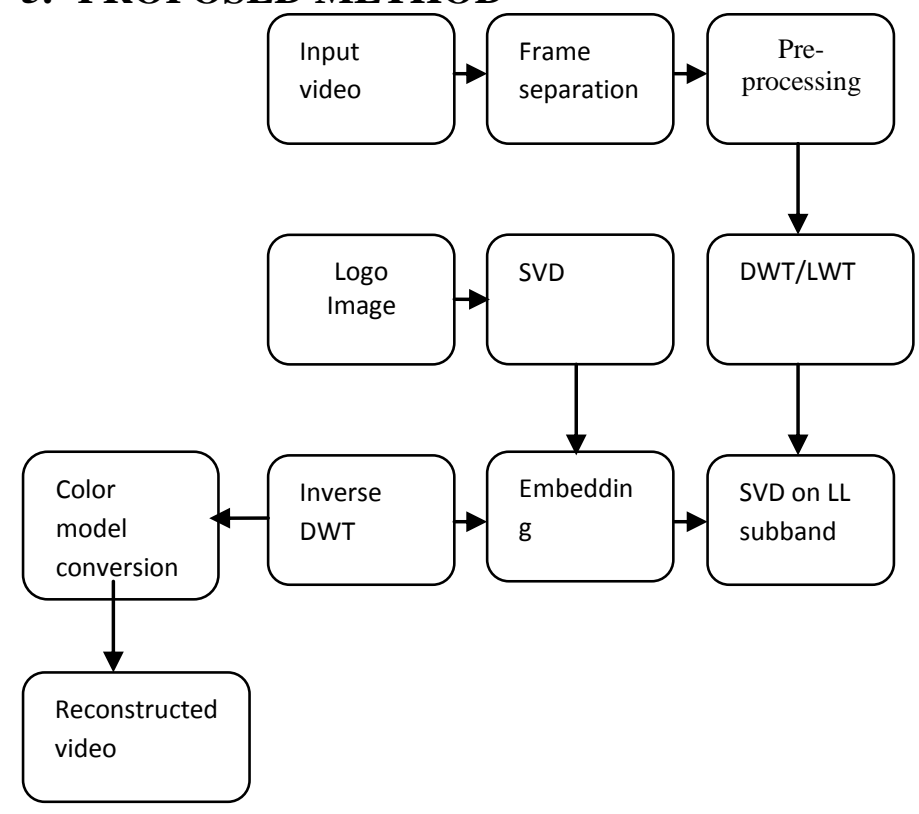

Fig. 1(a): Block diagram for Embedding Watermark

First of all, read the input video file in a MATLAB which is in a avi file format. Further modify that video file into the number of frame separation and applying pre-processing procedure on each frame. For pre-processing, Histogram Difference Method is apply on each frames of video and finds the shot change occurs in that video file. Now, applying DWT (Discrete Wavelet Transform)/LWT (Lifting Wavelet Transform) to the frames. Compute the data or information is in the form image/logo using $\operatorname{SVD}$ (Singular Value Decomposition).This is done for embedding the watermark procedure into the video file. Then performs inverse DWT/LWT transform to retains the watermark frame with the reconstructed video file having hidden image/logo.

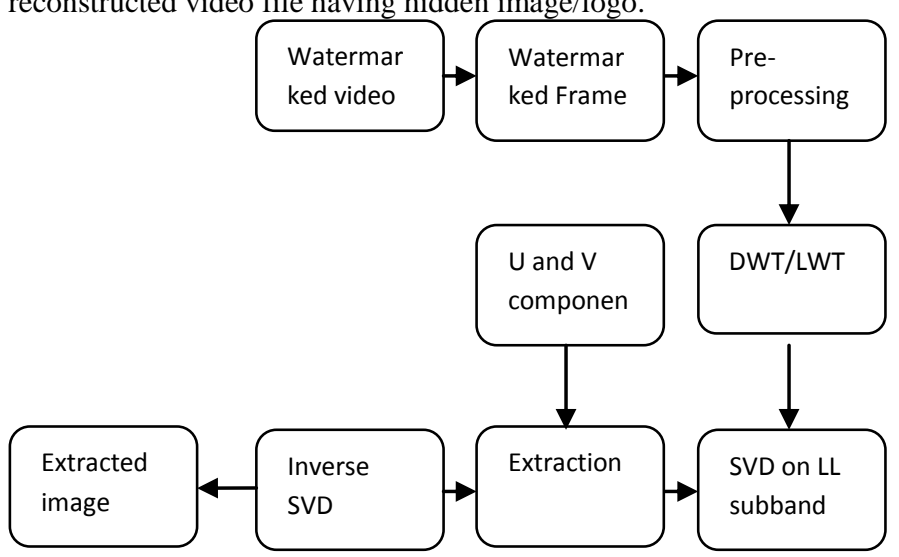

Fig. 1(b): Block diagram for Extracting Watermark

To extract the data or information which is in form of watermark image/logo into the frames from the reconstructed video, apply same transform DWT/LWT on SVD to retain the information such as to extract the image/logo. So, finally we get the original data/ information which we embedded secretly in a frame of video file. 


\section{IMPLEMENTATION DETAILS}

A MATLAB R2013a version is used to implement this methods and tested the performance of the DWT-SVD scheme and LWT-SVD scheme using an uncompressed avi file format video clip and a gray scale image is taken as the watermark image. To check the imperceptibility and strength of the watermarked video frame, we've calculated the PSNR(Peak Signal to Noise Ratio), CC(Co-relation coefficient) and SSIM(Structural Similarity Index ).

Mean Square Error (MSE), MSE is the value by averaging the squared intensity of the first (input) image and the resultant (output) image pixels as in (1).To find the PSNR value, we have to first calculate the value of MSE(Mean Square Error), which is given by:

$$
\mathrm{MSE}=\frac{1}{\mathrm{MN}} \sum_{\mathrm{i}=0}^{\mathrm{M}-1} \sum_{\mathrm{j}=0}^{\mathrm{N}-1}\left[\mathrm{~F}^{\prime}(\mathrm{i}, \mathrm{j})-\mathrm{F}(\mathrm{i}, \mathrm{j})\right]^{2}
$$

Here $\mathrm{F}$ is the original video frame and $\mathrm{F}^{\prime}$ is the watermarked video frame.

Imperceptibility (Transparency) of the watermark is estimated by PSNR (peak signal to noise ratio). If the PSNR is higher than we get the less distorted frame. In this way, the value of PSNR can be calculated using MSE by using the equation:

$$
\text { PSNR }=10 \log _{10} \frac{255^{2}}{\mathrm{MSE}}
$$

The peak signal-to-noise (PSNR) is that the ratio between a signal's most extreme power and also the power of the signal's noise. PSNR to live the standard of reconstructed pictures that are compressed. every pel (pixel) features a color value that may amendment once a picture is compressed then uncompressed. Signals will have a good dynamic vary, thus PSNR is typically expressed in decibels, that may be a ordered series.

Robustness may be a live of the immunity of the watermark against tries to get rid of or degrade it, internationally or accidentally. we have a tendency to measured the similarity between the initial cover frame and therefore the watermark embedded from image exploitation the correlation (CC), may be found as:

$$
\mathrm{CC}=\frac{\sum_{\mathrm{i}} \sum_{\mathrm{j}} \mathrm{W}(\mathrm{i}, \mathrm{j}) \mathrm{O}(\mathrm{i}, \mathrm{j})}{\sum_{\mathrm{i}} \sum_{\mathrm{j}}\left[\mathrm{W}(\mathrm{i}, \mathrm{j})^{2}\right]}
$$

Here $\mathrm{W}$ is that the Watermark image and $\mathrm{O}$ is that the original frame.

Correlation defines the similarity between same pel positions of the 2 pictures wherever one image is original watermark and alternative may be embedded

watermarked video frames.

The Structural Similarity Index (SSIM ) is that the mensuration of image quality supported an initial uncompressed or distortion-free pictures. SSIM is meant to enhance on ancient strategies like peak signal-to-noise (PSNR) and mean square error (MSE), that have proven to be inconsistent with human eye perception. SSIM may be a new paradigm for quality assessment, supported the hypothesis that the HVS is very custom-made for extracting structural data. The live of structural similarity compares native patterns of pel intensities that are normalized for physical property and distinction. The resultant SSIM list may be a decimal value between - one and one, and quality one is simply approachable on account of two indistinguishable arrangements of data. Numerically it's characterised,

$$
\operatorname{SSIM}=\frac{\left(2 \mu_{\mathrm{x}} \mu_{\mathrm{y}}+\mathrm{c}_{1}\right)\left(2 \sigma_{\mathrm{xy}}+\mathrm{c}_{1}\right)}{\left(\mu_{\mathrm{x}}^{2}+\mu_{\mathrm{y}}^{2}+\mathrm{c}_{1}\right)\left(\sigma_{\mathrm{x}}^{2}+\sigma_{\mathrm{y}}^{2}+\mathrm{c}_{2}\right)}
$$

Where,

$\mu_{\mathrm{x}}$ the traditional of $\mathrm{x}$;

$\mu_{\mathrm{y}}$ the traditional of $\mathrm{y}$;

$\sigma_{\mathrm{x}}^{2}$ the amendment of $\mathrm{x}$;

$\sigma_{\mathrm{y}}{ }^{2}$ the amendment of $\mathrm{y}$;

$\sigma_{\mathrm{xy}}$ the variance of $\mathrm{x}$ and $\mathrm{y}$;

$\mathrm{c}_{1}=\left(\mathrm{k}_{1} \mathrm{~L}\right)^{2}, \mathrm{c}_{2}=\left(\mathrm{k}_{2} \mathrm{~L}\right)^{2}$ two variables to balance out the division with frail denominator;

$\mathrm{L}$ is the dynamic vary of the pixel-values;

$\mathrm{k}_{1}=0.01$ and $\mathrm{k}_{2}=0.03$ by default.

The original input video(.avi) file-01 having size $8.90 \mathrm{MB}$ and 73 video frames, After applying histogram difference method, we are selected $60^{\text {th }}$ video frame and a gray scale watermark image of size $\left(185^{*} 202\right)$ are shown in the figure $1:(a),(b)$, (c).

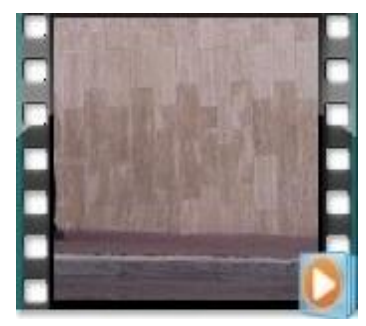

(a)

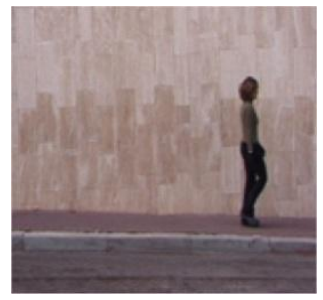

(b)

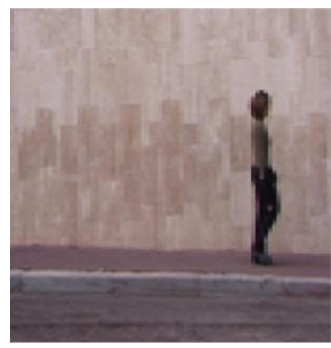

(d)

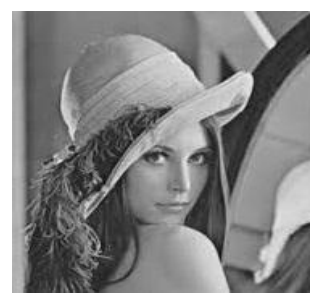

(c)

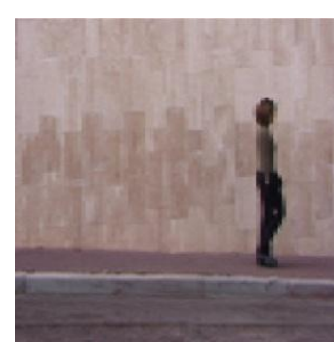

(e)
Fig. 5.1: (a) Input Video file-02 (b) Original Video Frame (c) Watermark Image (d) DWT Watermark Video Frame (e) LWT Watermark Video Frame 
The gray scale watermark image is used for embedding in the selected video frame and the embedded watermark with DWT and LWT schemes is shown in the figure 5.2: (d) and (e).From the above figures, it's clear that the LWT watermarked frames are highly imperceptible and robust than the DWT watermark frame. The embedded watermark is clear and very much similar to the original one (having the corelation coefficient 0.99-0.98).

\section{RESULTS}

The comparison of PSNR and SSIM values of both the watermarking schemes are mentioned in the following table:

Table 1. Comparison of MSE,PSNR and SSIM values of both schemes for 60th video frame

\begin{tabular}{|c|c|c|}
\hline \multirow{2}{*}{ Parameter } & $\begin{array}{c}\text { Embedding of } \\
\text { DWT } \\
\text { Watermark }\end{array}$ & $\begin{array}{c}\text { Embedding of } \\
\text { LWT } \\
\text { Watermark }\end{array}$ \\
\hline MSE & 4.543557 & 4.206289 \\
\hline PSNR & 41.556843 & 41.891813 \\
\hline CC & 0.993344 & 0.993478 \\
\hline SSIM & 0.938729 & 0.940695 \\
\hline
\end{tabular}

TABLE I shows that the PSNR, SSIM and CC values for the embedding of LWT watermark frame is more than the embedding of DWT watermark frame therefore the LWT \& SVD technique is a lot of strong and imperceptible than the DWT \& SVD technique.

\section{CONCLUSION AND FUTURE WORK}

In this paper, A video watermarking scheme using DWT \& SVD and LWT \& SVD methods have designed and implemented, The results (CC, PSNR and SSIM values) of LWT \& SVD scheme which are found to be quite satisfactory and are better in all respects than the DWT \& SVD scheme. In Video watermarking provides security mechanism against unwanted access to important information/logo by hiding in a video file such that the information remains invisible to the attacker and he is unable to distinguish between the hidden and the visible data. The proposed method will offer more protection and security to the video file along with high imperceptibility, robustness and low distortion of the watermark image with better results.

In future there is a scope of performing watermark extraction process to retrieve watermark image, to get the original watermark image which is embedded secretly in a video file. Also the time taken for embedding and extracting the watermark in both watermarking schemes are find out to analyse the time complexity The proposed scheme can also perform (on the same video clip) under various attacks like Cropping, Rotation, Salt \& Pepper Noise addition, Gaussian Noise addition, JPEG Motion 2000 Compression.

\section{ACKNOWLEDGMENTS}

It is a matter of nice pleasure and moment of large satisfaction on behalf of me to specific my profound feeling to my guide Prof. Aarti G. Ambekar, whose constant encouragement enabled to figure sky-high. His perpetual motivation, patience and glorious experience in discussion throughout progress of my work have benefited to extent, that is beyond.

\section{REFERENCES}

[1] Raghavendra K, Chetan K.R, "A Blind and Robust Watermarking Scheme with scrambled watermark for video authentication", IEEE International Conference, p.p . 1-6, December 2009.

[2] Sanjana Sinha, Prajnat Bardhan, Swarnali Pramanick, Ankur Jagatramka, “ Digital Video Watermarking using Discrete Wavelet Transform and Principle Component Analysis", International Journal of Wisdom based Computing, vol.1, August-2011.

[3] Tabassum, S.M. Mohidul Islam, “ A Digital Video Watermarking Technique based on identical frame extraction in 3-level DWT", IEEE, 2012.

[4] Himanshu Agarwal, Rakesh Ahuja, S.S. Bedi, "Highly Robust and Imperceptible Luminance Based Hybrid Digital Video Watermarking Scheme for Ownership protection”, I.J. Image, Graphics and Signal Processing, p.p. 47-52, November 2012.

[5] Souvik Sarkar, K. Senthilkumar, "A Highly secured Digital Watermarking Algorithm for Binary Watermark using Lifting Wavelet Transform and Singular Value Decomposition”, IET Third International conference on sustainable energy and intelligent system, p.p. 1-5, Dec 2012.

[6] Swanirbhar Majumder, Tirtha Sankar Das, Souvik Sarkar and SubirKumar Sarkar, "SVD and Lifting Wavelet based Fragile ImageWatermarking, ACEEE International Journal on Signal \& ImageProcessing, vol-2, no.1, Jan 2011.

[7] Pragya Agarwal, Arvind Kumar, Ankur Choudhary, "A Secure and Reliable Video Watermarking Technique",International Conference on Computer and Computational Sciences,IEEE, ISBN: 978-1-4799-1819$5 / 2015$.

[8] Prathik P, Rahul Krishna, Rahul Ajay Nafde, ShreeDarshan K, “ An Adaptive Blind Video Watermarking Technique based on SD-BPSO and DWTSVD", International Conference on Computer Communication and Informatics (ICCCI), January, 2013, Coimbatore, India.

[9] Bhavna Goel, Charu Agarwal, "An Optimized Uncompressed Video Watermarking Scheme based on SVD and DWT", IEEE International Conference, p.p. 307312, August 2013. 